\title{
Monitoring of bioelectrical and biomechanical signals in Taekwondo training: first insights
}

\author{
Bruno Amaro', Joel Antunes ${ }^{1}$, Pedro Cunha ${ }^{1}$, Filomena Soares ${ }^{1}$, Vítor \\ Carvalho $^{2}$, Hélder Carvalho ${ }^{3}$ \\ ${ }^{1}$ Dep.Industrial Electronics, Algoritmi Research Centre, University of Minho, Guimarães, \\ Portugal[a70785@alunos.uminho.pt; a61803@alunos.uminho.pt; id5514@alunos.uminho.pt; \\ fsoares@dei.uminho.pt] \\ ${ }^{2}$ School of Technology, IPCA, Barcelos \& Algoritmi Research Centre, Guimarães, University \\ of Minho, Portugal [vcarvalho@ipca.pt] \\ ${ }^{3}$ Dep.Textil Engineering, 2C2T Research Centre, University of Minho, Guimarães, Portugal \\ [helder@det.uminho.pt]
}

\begin{abstract}
Taekwondo is an Olympic combat sport that has gained much popularity in the last years. Portugal is not an exception to the substantial growth of this martial art. Currently there are about 4500 practitioners of this art affiliated in the national federation. Several have already produced excellent results not only in national but also in international competitions, recognized by the International Olympic Committee of Portugal, Taekwondo European Union and the World Taekwondo Federation (WTF). This work is a joint project between the University of Minho, Sporting Club de Braga Taekwondo section and the National Technical Team of the Portuguese Federation of Taekwondo. It is aimed at developing a system to analyse, monitor and quantify the athlete performance in real time. The developed system should be able to help the athlete improve his/her technique and performance by monitoring his/her heart rate, analyzing and identifying the technical movements in the training by comparing the various movements performed, as well as quantifying variables related to the athlete's performance. Visual cues for the heart rate and performance are indicated by LEDs. With this system, we propose a contribution to innovation and development in Taekwondo training.
\end{abstract}

Keywords: Bioeletric, biomechanic, martial arts, motion analysis, Taekwondo.

\section{Introduction and objectives}

Ubiquitous computing is a fast-growing field with applications in many areas. Sports is one of the fields where this growth is most visible. Today it is possible to 
monitor athletes in various aspects, for example, by tracking and recognizing movements, measuring forces and accelerations, quantifying the effort made and many other functionalities.

Taekwondo is a full contact martial art that mainly uses leg movements to try to attack the torso and the opponent's head [1]. There are also some arm movements, but they can only be directed against the opponent's torso. Taekwondo has become a popular sport and all the tools able to improve athlete performance are valuable.

This project studies existing training equipment and automatic scoring systems for competition to understand weaknesses and try to create an improved product. As new functionalities, heart rate acquisition was introduced, movements are analyzed using Inertial Measurement Units (IMUs) and the impact is measured by flexible piezoresistive sensors. The objective is to integrate these functionalities into the training suits, making them a wearable system with which it will be possible for the athlete to improve training and increase performance. Various visual indications will be given by light emitting diodes (LEDs), including heart rate. In this way, the athlete's performance and status can be easily followed. It is possible that the system evolves to a new type of scoring system for competition, considering that it is known that current commercial systems produce some errors and do not detect all impacts and movements[2].

The project intends to meet the following requirements and restrictions:

- Real-time measurement;

- Develop impact detection based on integrated sensor signals;

- Analysis of the trajectories based on measurement by IMUs;

- Development of an interface for displaying data, on the equipment (light) and on a mobile device/computer;

- Adequate range of communication;

- Positioning of components ensuring their safety and that of the athlete;

- The athlete's comfort.

This paper is organized in six sections. The second section describes the state or art of systems used in the points scoring of Taekwondo; the third section presents the methodology and the solution developed requirements and architecture; the fourth section describes the materials used; the fifth section shows some experimental results; and, finally, the sixth section enunciates some conclusions and future work developments.

\section{State of the art}

Taekwondo is a traditional Korean martial art that literally means "the way of kicking and punching". The most important movements of Taekwondo are the combination of kick movements, but hands can also be used to strike the opponent. 
It was in the early 20th century that Taekwondo became the dominant martial art practiced in Korea. As a result, Taekwondo became Korea's national martial art and was promoted internationally. The World Taekwondo Federation (WTF) was founded in 1973, and became the worldwide legitimate responsible of the sport. In the 1988 Seoul Games, Taekwondo made the first demonstration in an Olympic event. It was in 2000, in the Games of the XXVII Olympiad in Sydney, that Taekwondo became an official medal sport [3].

Because of the sport's popularity, the demand to assure accuracy and fairness in judging has increased. This demand lead to several rule changes and created an opportunity for the use of technology. Technology appears as a solution to solve the inherent judging problems. As a result, the PSS (Protector Scoring Systems) were created. The most used PSS systems are the Daedo products, but they are not flawless.

Taekwondo is a full-contact sport that uses both head and body protectors. In competitions, there are two athletes in different colours that fight in a 12-meterwide square padded mat and three judges placed around the mat in a triangular shape, with a handset to score valid points.

The Daedo system (Fig. 1) is composed of [4]:

- Electronic protector, which has the function of protecting the athlete and help scoring;

- Electronic Foot Protector, which has 5 different sensors located in strategic points around the foot, allowing to score valid kicks;

- Electronic Head Gear, which scores points in all valid areas of the head, including the face;

- Transmitter, which sends the signals of the detected impacts from the electronic protector to the computer;

- Receiver, which receives the signal from the transmitter;

- Judge Scoring Box, which can be pressed by the referee during a match to assign points that were not automatically assigned by the system.

As described above, the Daedo system has several components related to scoring. However, presently there is no system capable of acquiring vital signals of the athlete, nor doing motion analysis using sensors.

Considering that reality, in [5], the authors present an impact measurement technique which integrates a "non-invasive" sensor system into a Taekwondo body protector. The system uses Bluetooth to transmit sensor readings to a computer for analysis. Impact is measured using thin film piezoresistive force and pressure sensors, besides MEMS (Micro-Electro-Mechanical Systems) accelerometers. In the obtained results, a limitation of the Bluetooth bandwidth was found, limiting the amount of information that could be transmitted. The authors also refer to the need of a strategy to manage that information. This shows that there is a need to study and develop robust systems for this data acquisition and analysis task. 
Chi et al [2] have succeeded in integrating wireless scoring devices as part of the competition environment. Their system uses piezoelectric force sensors and transmits signals wirelessly to enable the detection of significant impacts delivered to the competitor's body. In their conclusions, it is stated that this objective is attained with success.

\section{Methodology and definition of a solution}

The methodology followed for the development of the proposed system was divided in the following steps:

- First, existing sensors were researched to find a solution for each functionality required. More specifically, the sensors studied were piezoresistive impact/force sensors, IMUs and sensors for heart rate measurement.

- Second, after the definition of the sensors, their integration and location in the practitioner's equipment was determined. Preliminary tests with the sensors were carried out using an Arduino Uno and LabVIEW software. Then, the sensor choice and location were validated.

- Third, based on the information gathered in the previous task, the hardware requirements in terms of signal acquisition and data communication were defined and a system architecture was delineated, as shown in Fig. 1.

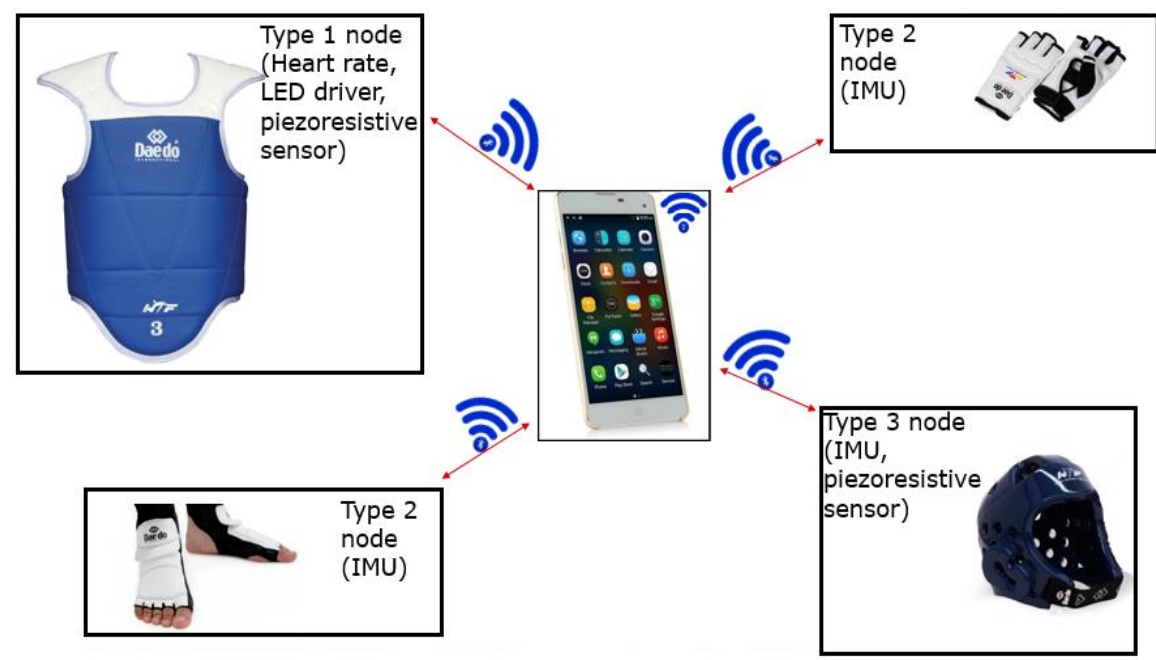

Fig. 1 System architecture (Source of equipment images: Daedo [6])

There are three types of nodes. Type 1 nodes include the acquisition of heart rate and piezoresistive sensor signals for impact measurement, but also expose the heart 
rate through programmable LEDs. Type 2 consists of the acquisition of IMU signals and type 3 nodes acquire only impact signals.

The LEDs in the body protector display the heart rhythms of the athlete through a colour code, with each of the heart rate zones being shown by a specific colour; other information such as impacts or valid hits could be briefly superimposed on the heart rate information.

Communication is implemented using Bluetooth in a star configuration, with the mobile phone being the central node receiving all the information.

In the next stage, hardware and firmware for the several types of sensor nodes, as well as transmission to a mobile device were developed and prototyped.

The fifth step is the development of an app for a mobile device based on Android. A simple and user-friendly interface is a key point in this matter, given that the coaches must pay attention to both the information displayed on the app, on the LEDs as well as, evidently, on the trainee.

Currently, and for testing and development purposes, the application displays three types of graphs: one with IMU data, another with impact data and another with the data of the heart rate sensor.

After a first prototype is available and tested, a phase of data analysis begins. In this phase, actual data from training sessions will be acquired and analysed with the purpose of extracting the relevant signal features that will allow identifying trajectories, techniques, and impacts.

Finally, a database will be structured to store the relevant information resulting from the signal processing techniques in an appropriate manner. The objective is to store correct movement data, to compare the practitioners' movements with the "gold standards" stored in the database.

\section{Materials}

Based on the system requirements and the architecture previously defined, hardware was selected to implement a prototype.

Node 1 prototype, integrated in the chest protector, uses an Arduino board as data acquisition device and a HC-06 Bluetooth module. Future versions could use smaller boards (Arduino Mini or the tinyTILE - Intel Curie module based boards, which already integrates Bluetooth Low Energy and an IMU).

The piezoresistive sensor [7] (Fig. 2) used is manufactured at the University of Minho and is based on a piezoresistive conductive polyethylene film. It is composed of the transducer piezoresistive film and two electrodes, one on each side of the piezoresistive film, allowing the electrical connection. The electrical resistance of this assembly varies according to the force applied on it. 


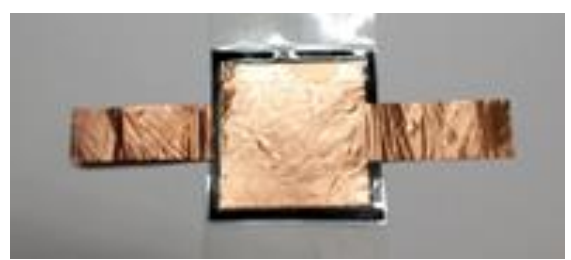

Fig. 2 Piezoresistive sensor [7]

Signal conditioning for the piezoresistive sensor is achieved using the following circuit presented in Fig. 3.

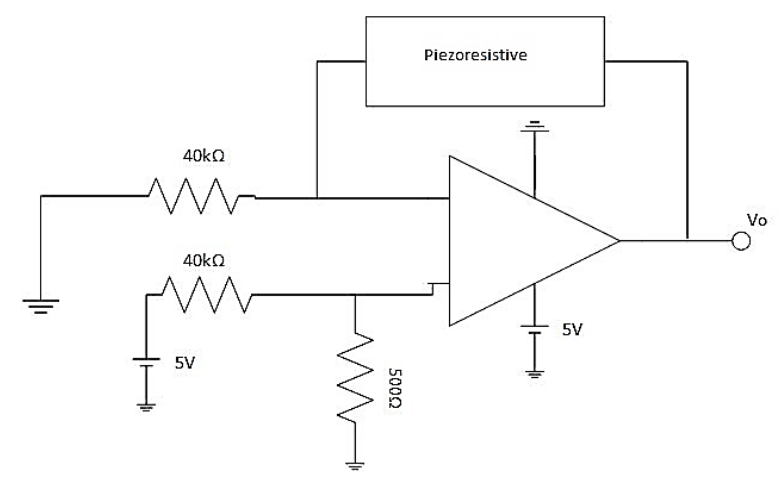

Fig. 3 Piezoresistive sensor signal conditioning circuit. Output voltage Vo inversely proportional to sensor resistance

The visual clues are displayed by programmable WS2812 5050 RGB LEDs, often known as "Neopixels".

For the prototype, heart rate was measured using a Polar T34 cheststrap and WearLink + compatible receiver. The receiver delivers a pulse for each R-wave of the ECG signal. Heart rate is computed by the microcontroller measuring the interval between two pulses. The final implementation is intended to use textile electrodes embedded in a shirt, as described in [8].

Node 2 prototype is used in the athlete's gloves and foot protector. It also uses the Arduino board as data acquisition device, combined with the HC-06 Bluetooth module and an MPU 6050 breakout board for IMU data.

The node 3 prototype is like nodes 1 and 2. It is used in the headgear and includes piezoresistive sensors for acquiring impacts.

\section{Experiments and Results}

For the development of the project, the following tasks have been carried out so far and some results will be shown in this section, namely: 
- Acquisition of IMU, impact and heart rate signals;

- Transmission of signals via Bluetooth to a computer with LabVIEW, for first tests;

- Transmission of all sensor data via Bluetooth to a mobile phone;

- Implementation and test of the display using the programmable LEDs;

- Prototype Android app.

\section{Piezoresistive sensor}

Fig. 4 shows a series of impacts on the piezoresistive sensor. The developed system only considers a valid impact when the applied force exceeds a defined limit, avoiding reading small touches that are not impacts.

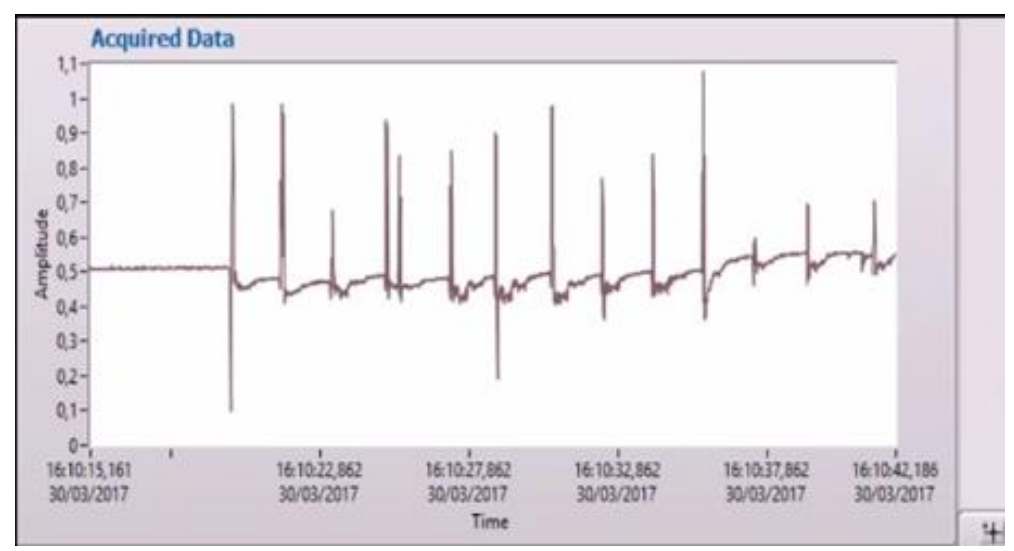

Fig. 4 Impact sensor signal for a series of punches

\section{$I M U$}

Two different hand attack techniques, namely a forefist and a hook, were executed, without impact, by a Taekwondo athlete with a node 2 prototype attached to his hand, as shown in Fig.5. The resulting gyroscope signals can be seen in Fig.6. 


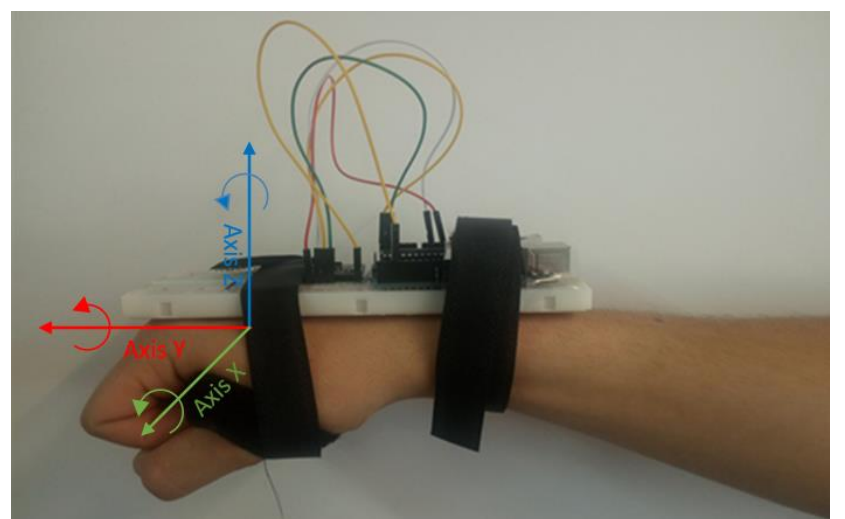

Fig. 5 Setup of the node for acquisition of IMU data during a punch
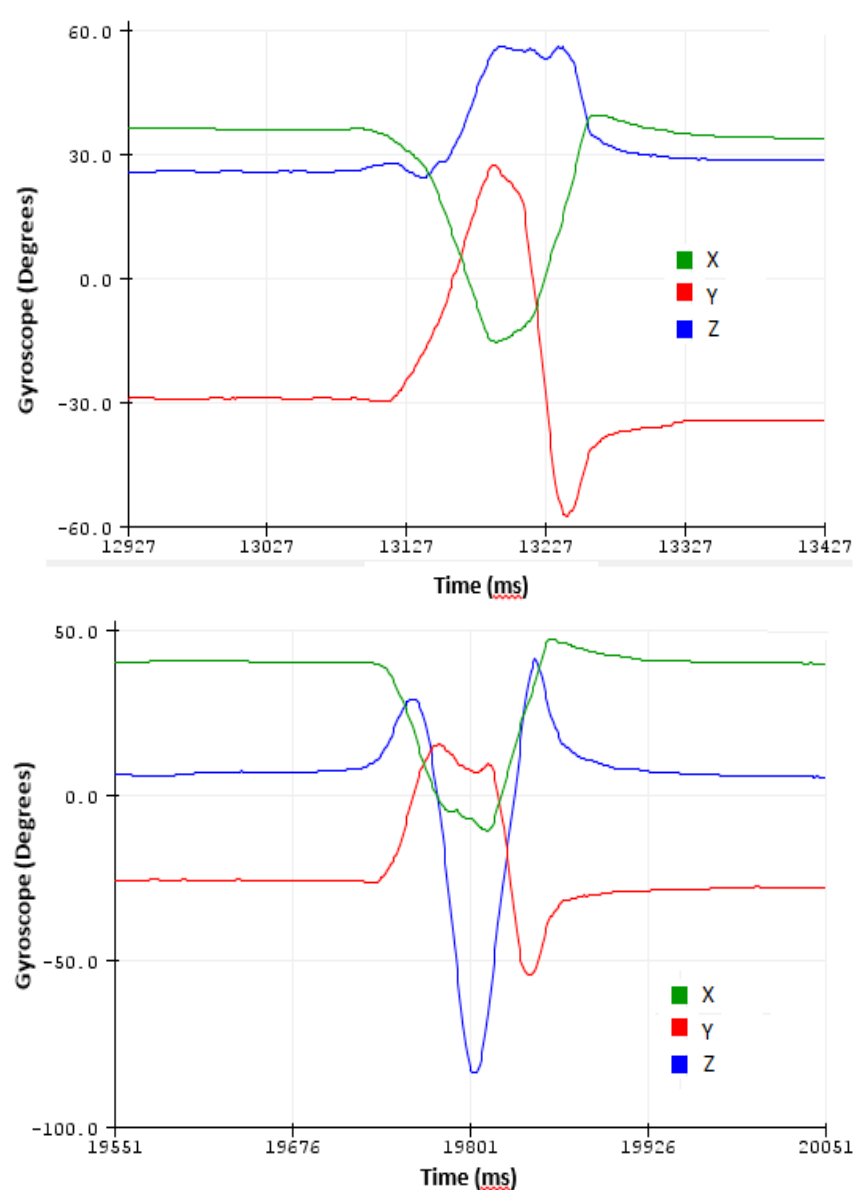

Fig. 6 Gyroscope signals for forefist (upper) and hook (lower) punches 
As can be seen, the signals clearly show the differences between the two techniques. In the first graph, where the forefist technique was used, the most significant rotation is around the $\mathrm{Y}$ axis, whilst the hook produces a higher rotation signal around the $\mathrm{z}$-axis - about 90 degrees, as expected.

\section{Heart rate sensor}

The heart rate receiver provides a TTL-compatible output that was connected to an interrupt of the Arduino board. Timing between two consecutive pulses allows computation of the heart rate. The heart beat value is updated every 5 heart beats, being the average of the 5 intervals. It has been found before [8] that movement causes false or missed pulses. To avoid false computations, a value is rejected if the current measurement differs more than $15 \%$ from the last one.

\section{Android App}

Finally, the last task carried out so far is the development of the application, not yet fully completed. It is possible to pair and connect to the external nodes, and some basic signal graphs can be displayed.

\section{Conclusions and future work}

The development proposed in this paper is a challenging task because it combines the measurement and interpretation of different variables involved in Taekwondo training and competition. Martial arts are no exception in the current trend of acquiring and analyzing "big data" with the associated difficulties.

The system acquires movement signals that we expect will be most useful in quantifying performance and providing a tool to improve technique. Impact signals combined with IMU can mean a more reliable way of scoring, whilst heart rate measurement enables monitoring of the athlete's physical state.

Future work will involve the acquisition and processing of real data, such as movements performed by expert practitioners, to be used as gold standards, and movements performed by athletes to be compared with those standards. This comparison will enable the specification of the signal features that can quantify the athlete performance. A database for recording of the individual athlete's performance and workout data will provide a tool to track the athlete's improvement and health status over time. 


\section{Acknowledgements}

This work is financed by FEDER funds through the Competitivity Factors Operational Program - COMPETE and by national funds through FCT - Foundation for Science and Technology within the scope of the project POCI-01-0145-FEDER007136. Pedro Cunha thanks FCT for PhD scholarship SFRH/BD/121994/2016.

Special thanks to Coach Joaquim Peixoto, as well as Sport Club de Braga Taekwondo section and to the national team, for allowing us to use their training site, as well as seeing/participating in training to test the developed system, and in general, for all the cooperation provided.

\section{References}

[1] Idesporto.pt. (2017). IPDJ, I.P. website[online] Available at: http://www.idesporto.pt/conteudo.aspx?id=103 [Accessed Mar. 2017].

[2] E. H. Chi, J. Song, and G. Corbin, Killer app of wearable computing: wireless force sensing body protectors for martial arts, in UIST 2004: Proceedings of the 17th annual ACM symposium on User interface software and technology, New York, NY, USA: ACM, 2004, pp. 277 285 .

[3] International Olympic Committee. (2017). Taekwondo Equipment and History - Olympic Sport History. [online] Available at: https://www.olympic.org/Taekwondo-equipment-and-history [Accessed Mar. 2017].

[4]Daedo International (2013) TK-STRIKE User Guide [Online]. Available: https://sw3362.smartweb-static.com/upload_dir/docs/Manual-TK-Strike-Truescore-2014.pdf. [Accessed: Mar. 2017].

[5] J.I. Cowie, J.A. Flint, A.R. Harland, Wireless Impact Measurement for Martial Arts, in M. Estivalet and P. Brisson, The Engineering of Sport 7. Guildford: Springer London, 2008, pp 231-237.

[6] Protectores - Taekwondo, Daedo.com, 2017. [Online]. Available:

https://www.daedo.com/taekwondo/protectors/. [Accessed: 20- Jun- 2017].

[7] H Carvalho, Y Yao and L M Gonçalves, Flexible force sensors for e-textiles, Proceedings of Autex- World Textile Conference, 29-31 May 2017, Corfu, Greece.

[8] Lage, J., Catarino, A.P., Carvalho, H., Rocha, A.M., Smart Shirt with Embedded Vital Sign and Moisture Sensing, Proceedings of SPWID 2015: The First International Conference on Smart Portable, Wearable, Implantable and Disability-oriented Devices and Systems, Iaria, ISBN: 978-1-61208-446-6 Brussels, Belgium, June 21 - 26, 2015. 